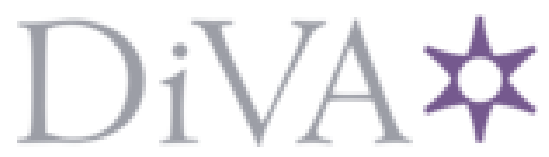

http://www.diva-portal.org

This is the published version of a paper presented at IEEE 15th International Conference on Environment and Electrical Engineering, June 10-13, Rome, Italy.

Citation for the original published paper:

Ur-Rehman Malik, N., Almas, M., Vanfretti, L. (2015)

Challenges of Real-Time Parameter Estimationof a DFIG using Synchrophasors.

In:

N.B. When citing this work, cite the original published paper.

Permanent link to this version:

http://urn.kb.se/resolve?urn=urn:nbn:se:kth:diva-170844 


\section{Challenges of Real-Time Parameter Estimation of a DFIG using Synchrophasors}

\author{
N. ur Rehman Malik, and M. S. Almas \\ School of Electrical Engineering, \\ KTH Royal Institute of Technology, \\ Stockholm, Sweden. \\ Email: (nurmalik,msalmas@kth.se)
}

\author{
L. Vanfretti \\ KTH Royal Institute of Technology, Stockholm, Sweden \\ R\&D Department, Statnett SF, Oslo, Norway. \\ Email: luigiv@kth.se, luigi.vanfretti@ statnett.no
}

\begin{abstract}
This paper explores the challenges faced during the utilization of synchrophasors received from PMUs in order to estimate the parameters of a DFIG in a real-time. PMUs are installed at the stator and rotor terminals of an 11-KVA DFIG, in order to provide high resolution synchrophasor data at a reporting rate of $50 \mathrm{msgs} / \mathrm{sec}$. These synchrophasors are processed in real-time using an embedded controller in order to measure and estimate the internal parameters of the generator, i.e., magnetization and leakage inductances. Closed-loop variable speed drive, active power and reactive power controls are implemented for the DFIG. This paper discusses the laboratory setup, control and parameter estimation experiments carried out on the DFIG. The testing process, challenges faced, and overall experimental findings are also presented.
\end{abstract}

Index Terms-DFIG, parameter estimation, PMUs, synchrophasors, vector control, wind power.

\section{INTRODUCTION}

Utilization of renewable energy sources (such as wind and the sun) for electricity production has risen rapidly in the last few years, leading to an increasing number of wind and photovoltaic plants being integrated into the power grid. This increase of variable generation sources poses difficulties for system and plant operators for dispatch, operation, and control of these resources. Hence, new means for control of renewable sources are attractive to manage uncertainties in their operation.

The identification of the parameters of wind generators are important for implementing various dynamic controls. This is because the inductances of the generator changes with load and, in fact, saturate at peak loads. Furthermore, resistances of the generator grow with increases in the temperature due to large conduction currents. Therefore, continuous evaluation of generator parameters may assist in its condition monitoring and, in the worst case, it can provide information of the generator faults, (e.g., insulation failures, winding inter-turn short-circuits, etc.).

The doubly-fed induction generator (DFIG) is one of the most commonly used machines for wind power generation. These generators employ a partially-rated converter, having power ratings of $\pm 30 \%$ of the generator for $\pm 25 \%$ speed ranges [1], [2]. This is an obvious advantage in terms of cost savings at higher power levels, since the power rating of the power electronic converter is only approximately $0.65 \mathrm{MW}$ for a 2 MW generator. In the past, different methods have been employed in order to estimate the internal parameters of a DFIG. One of the most common methods are offline tests [3], such as no-load and locked-rotor tests. The parameters measured by such tests can be different from those seen during operation, due to varying magnetic saturation and temperature effects once the generator is connected to the grid. Moreover, properties of the generator, (e.g., magnetic characteristics of the iron core and the winding insulation strength) change with time. As a result, online parameter estimation becomes necessary. In addition, it will help in the real-time calibration and validation of the mathematical models of the wind farms that are being used for stability studies and insulation condition monitoring, and may assist transmission system operators (TSOs) and wind-farm owners to continuously monitor the dynamic performance of their units [4].

Since the last decade, installation of synchrophasor technology (also known as Phasor Measurement Units (PMU's)) has increased. The technology is being used to improve grid performance, through timely detection of the faults and instabilities [5]. The greatest advantage of the synchronized phasors is that, it provides high resolution time-tagged voltage and current phasors. Thus, continuous availability of the measurements from the different locations may be exploited for control of the renewable generation [6]. The main purpose of this paper is to explore the challenges and limitations of utilizing synchrophasor measurements, in real-time, for internal parameter estimation of a DFIG system. The experiments are conducted on an 11-KVA DFIG laboratory test-bench coupled with PMUs. The PMUs are installed both at the rotor and stator terminals of the DFIG. The novel approach adopted for this experiment, lessons learnt, limitations discovered, recommendations, and a road-map for future work in this domain is presented and discussed. Finally, the need for development of Multi-Frequency or Dynamic PMU units to provide better estimation of the parameters of the sub-components of the grid by exploiting the local and remote synchrophasors is highlighted.

The paper is organized as follows. Section II and Section III presents the electrical model and vector control of the DFIG, respectively, whereas Section IV and Section V presents the 
experimental setup, and result analysis, respectively. Finally, discussions and conclusions are presented.

\section{DFIG DYNAMIC MODEL}

The dynamic model of the generator is formulated in the $d q$ frame [7]. The model is used to derive current controllers, used for generator control. The electrical dynamics of the generator are:

$$
\begin{aligned}
& \frac{d \psi_{\mathrm{sd}}}{d t}=v_{\mathrm{sd}}-R_{\mathrm{s}} i_{\mathrm{sd}}+\omega_{\mathrm{s}} \psi_{\mathrm{sq}} \\
& \frac{d \psi_{\mathrm{sq}}}{d t}=v_{\mathrm{sq}}-R_{\mathrm{s}} i_{\mathrm{sq}}-\omega_{\mathrm{s}} \psi_{\mathrm{sd}} \\
& \frac{d \psi_{\mathrm{rd}}^{\prime}}{d t}=v_{\mathrm{rd}}^{\prime}-R_{\mathrm{r}}^{\prime} i_{\mathrm{rd}}^{\prime}+\left(\omega_{\mathrm{s}}-\omega_{\mathrm{rm}}\right) \psi_{\mathrm{rq}}^{\prime} \\
& \frac{d \psi_{\mathrm{rq}}^{\prime}}{d t}=v_{\mathrm{rq}}^{\prime}-R_{\mathrm{r}}^{\prime} i_{\mathrm{rq}}^{\prime}-\left(\omega_{\mathrm{s}}-\omega_{\mathrm{rm}}\right) \psi_{\mathrm{rd}}^{\prime} \\
& \psi_{\mathrm{sd}}=L_{\mathrm{sl}} i_{\mathrm{sd}}+L_{\mathrm{ms}}\left(i_{\mathrm{sd}}+i_{\mathrm{rd}}^{\prime}\right) \\
& \psi_{\mathrm{sq}}=L_{\mathrm{sl}} i_{\mathrm{sq}}+L_{\mathrm{ms}}\left(i_{\mathrm{sq}}+i_{\mathrm{rq}}^{\prime}\right) \\
& \psi_{\mathrm{rd}}^{\prime}=L_{\mathrm{rl}}^{\prime} i_{\mathrm{rd}}^{\prime}+L_{\mathrm{ms}}\left(i_{\mathrm{sd}}+i_{\mathrm{rd}}^{\prime}\right) \\
& \psi_{\mathrm{rq}}^{\prime}=L_{\mathrm{rl}}^{\prime} i_{\mathrm{rq}}^{\prime}+L_{\mathrm{ms}}\left(i_{\mathrm{sq}}+i_{\mathrm{rq}}^{\prime}\right)
\end{aligned}
$$

where $R_{\mathrm{s}}$ and $R_{\mathrm{r}}^{\prime}$ are the the stator and rotor resistances of the DFIG, respectively, whereas $L_{\mathrm{ms}}, L_{\mathrm{sl}}$, and $L_{\mathrm{rl}}^{\prime}$ are the magnetization, stator leakage, and rotor leakage inductances of the DFIG, respectively. $\psi_{\mathrm{sd}}, \psi_{\mathrm{sq}}, \psi_{\mathrm{rd}}^{\prime}$, and $\psi_{\mathrm{rq}}^{\prime}$ are the stator $d q$-fluxes and rotor $d q$-fluxes, respectively, whereas $i_{\mathrm{sd}}, i_{\mathrm{sq}}$, $i_{\mathrm{rd}}^{\prime}$, and $i_{\mathrm{rq}}^{\prime}$ are the stator $d q$ currents and rotor $d q$ currents, respectively. $\omega_{\mathrm{s}}$ and $\omega_{\mathrm{rm}}$ are the stator frequency and rotor speed (in $\mathrm{rad} / \mathrm{s}$ ), respectively.

\section{Vector Control of the DFIG}

The vector control for variable speed control of the DFIG is implemented using the dSpace platform. The control is implemented in a synchronous $d q$ frame, where the $d$-axes is aligned with the grid flux and the $q$-axes is in quadrature. In this way, independent control of the active- and reactivepowers is realized.

\section{A. Phase-Locked Loop}

In order to synchronize the generator with the grid and deliver power to the network, it is necessary that the grid flux angle and its magnitude is estimated with considerable accuracy. In order to do so, a phase-locked loop (PLL) algorithm proposed in [8], is implemented, as

$$
\begin{array}{r}
\frac{d \hat{\omega}_{1}}{d t}=\gamma_{1} \epsilon, \quad \frac{d \hat{\theta}_{1}}{d t}=\hat{\omega}_{1}+\gamma_{2} \epsilon, \\
\epsilon=K\left[m\left(\theta_{1}-\hat{\theta}_{1}\right)\right], \quad \gamma_{1}=\rho^{2}, \quad \gamma_{2}=2 \rho,
\end{array}
$$

where $\epsilon$ is the error between the actual position $\left(\theta_{1}\right)$ and the estimated position $\left(\hat{\theta}_{1}\right)$ of the grid-flux vector [8]. $K$ and $m$ are the application dependent constants [8], whereas $\rho$ defines the bandwidth of the PLL.

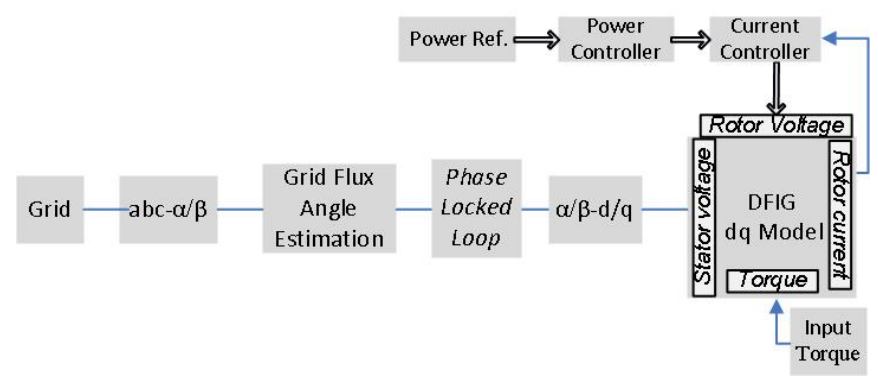

Fig. 1. Doubly-fed induction generator (DFIG) control scheme.

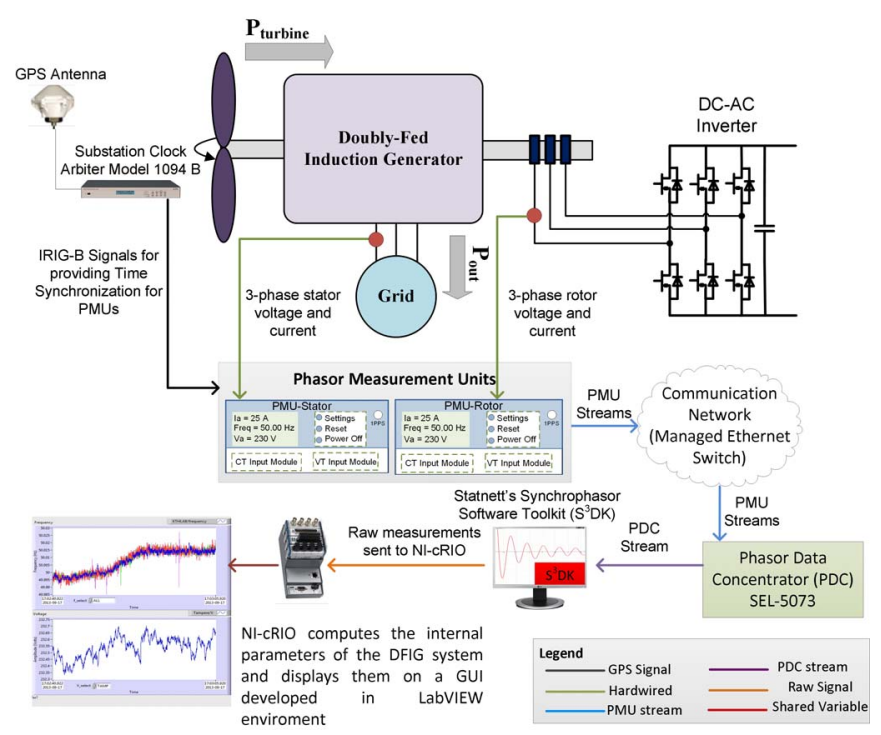

Fig. 2. Schematic of the experimental setup showing the coupling of PMUs at both the stator and rotor terminals of the DFIG.

\section{B. DFIG Rotor Current Controllers}

In order to control the active- and reactive-powers being delivered to the grid, rotor current controllers in the $d q$ reference frame are designed and implemented [9]. Because the $d$-axes is aligned with the grid-flux, the $d$-component of the rotor current is used to vary the reactive power, and therefore, the DFIG's output voltage. Furthermore, the $q$-component of the rotor current is used to change the active power, which is produced by the generator and delivered to the grid. The current controller of the generator is given by

$$
\begin{gathered}
F_{\mathrm{c}}(s)=\frac{\alpha_{\mathrm{c}}}{s} G_{\mathrm{c}}^{-1}(s)=k_{\mathrm{pc}}+\frac{k_{\mathrm{ic}}}{s} \\
k_{\mathrm{pc}}=\alpha_{\mathrm{c}}\left(L_{1}+L_{2}^{\prime}\right), \quad k_{\mathrm{ic}}=\alpha_{\mathrm{c}}\left(R_{1}+R_{2}^{\prime}\right),
\end{gathered}
$$

where $G_{\mathrm{c}}(s)$ is the transfer function, which represents the DFIG (see Section II), whereas $\alpha_{\mathrm{c}}$ is the bandwidth of the closed current-control loop. $k_{\mathrm{pc}}$ and $k_{\mathrm{ic}}$ are the proportional and integral gains, respectively.

The control scheme of the DFIG is shown in Fig. 1. 


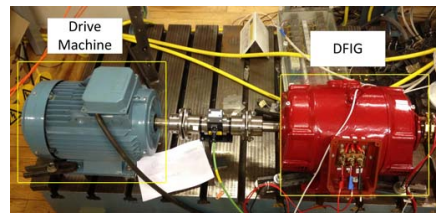

(a)

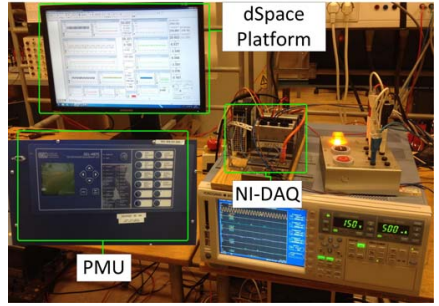

(b)

Fig. 3. Experimental test bench in the laboratory: (a) DFIG and the drive machine, (b) dSpace graphical interface, PMU, and the NI data acquisition system.

\section{DFIG Stator and Rotor Powers}

The stator active and reactive powers of the DFIG are expressed [7], as

$$
\begin{aligned}
P_{\mathrm{s}} & =\frac{3}{2}\left[v_{\mathrm{sd}} i_{\mathrm{sd}}+v_{\mathrm{sq}} i_{\mathrm{sq}}-R_{\mathrm{s}}\left(i_{\mathrm{sd}}^{2}+i_{\mathrm{sq}}^{2}\right)\right] \\
Q_{\mathrm{s}} & =\frac{3}{2}\left(v_{\mathrm{sq}} i_{\mathrm{sd}}-v_{\mathrm{sd}} i_{\mathrm{sq}}\right)
\end{aligned}
$$

whereas the rotor active and reactive power of the DFIG are represented [7], as

$$
\begin{aligned}
P_{\mathrm{r}} & =\frac{3}{2}\left[v_{\mathrm{rd}} i_{\mathrm{rd}}+v_{\mathrm{rq}} i_{\mathrm{rq}}-R_{\mathrm{r}}^{\prime}\left(i_{\mathrm{rd}}^{2}+i_{\mathrm{rq}}^{2}\right)\right] \\
Q_{\mathrm{r}} & =\frac{3}{2}\left(v_{\mathrm{rq}} i_{\mathrm{rd}}-v_{\mathrm{rd}} i_{\mathrm{rq}}\right) .
\end{aligned}
$$

\section{EXPERIMENTAL SETUP}

The experimental setup is depicted in Fig. 2, whereas Fig. 3 shows the experimental test-bench in the laboratory. PMUs are coupled at both the stator and rotor terminals of an 11-KVA DFIG. Time synchronization signals using IRIG-B are provided to the PMUs using a substation clock (Arbiter 1094B), which has an accuracy of \pm 100 ns. The PMUs, stream out synchrophasors for both the rotor and stator three-phase voltages and currents, in the C37.118.1 - 2011 format [10], at a reporting rate of $50 \mathrm{msgs} / \mathrm{s}$. These PMU streams are time-aligned and concentrated by a Phasor Data Concentrator (PDC) $(S E L-5073)$. A PDC output stream is configured to provide only the positive-sequence voltages and currents for both the stator and rotor. This PDC stream is received in a PC and are processed using Statnett's Synchrophasor Software Development Kit $\left(S^{3} \mathrm{DK}\right)$ [11]. This toolkit unwraps the PDC streams, and provides access to the raw phasors, analogs, and digital quantities, wrapped inside the $C 37.118 .1$ - 2011 data packet. These raw measurements are received in the National Instrument-based Compact Reconfigurable I/O Controller (NIcRIO), in order to utilize them for estimating the internal parameters of the DFIG. Once the parameters are estimated, these values are graphically shown as waveforms in a graphical user interface (GUI), developed in LabVIEW. The cRIO is chosen over the PC so that the controls and protection functions for the DFIG can be implemented in this hardware in the future.

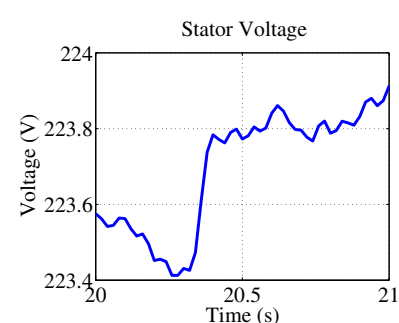

(a)

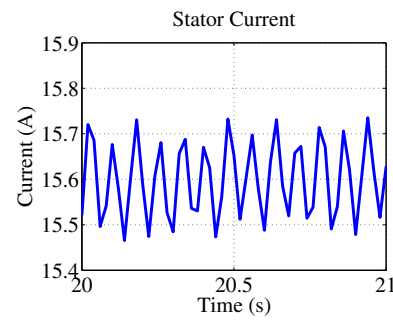

(c)

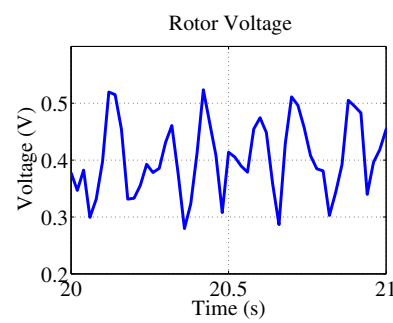

(e)

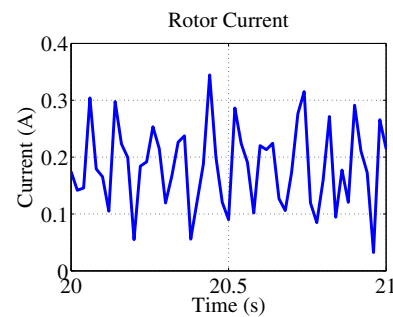

(g)

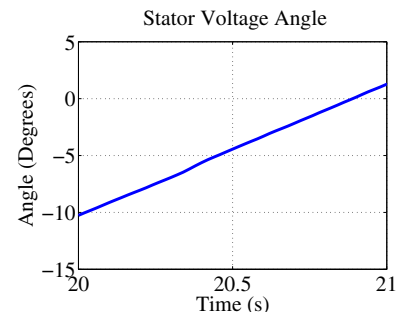

(b)

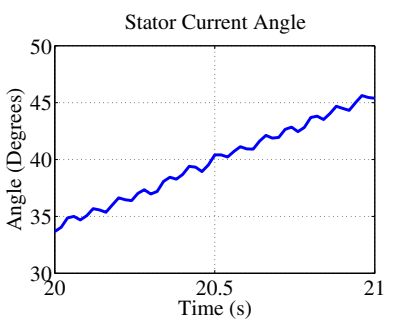

(d)

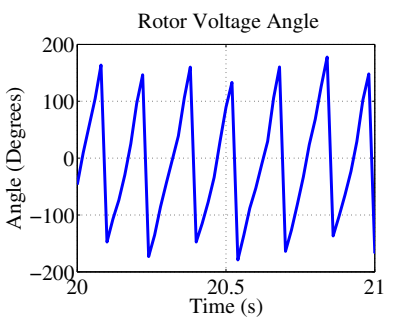

(f)

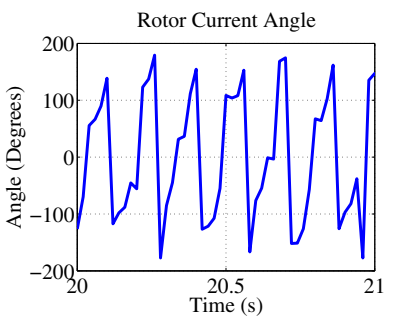

(h)
Fig. 4. Synchrophasor measurements of the DFIG from the PMUs, (at the speed of $1700 \mathrm{rpm}$ and $9 \mathrm{~kW}$ operation). The waveforms show the: (a) stator voltage magnitude, (b) stator voltage angle, (c) stator current magnitude, (d) stator current angle, (e) rotor voltage magnitude, (f) rotor voltage angle, (g) rotor current magnitude, and (h) rotor current angle.

\section{Results, OBservations, And AnAlysis}

\section{A. Experiment with the PMU's Connected to the Stator and Rotor Terminals}

In order to analyze the synchrophasors computed at the stator and rotor by the PMUs (according to the experimental setup in Fig. 2 and Fig. 3), a test scenario is executed. In this scenario, the DFIG is connected to the grid, which generates $9 \mathrm{KW}$ of active power. The DFIG's speed is maintained at $1700 \mathrm{rpm}$ via a speed controller. The synchrophasors computed by both PMUs are shown in Fig. 4. The stator is connected to the grid, therefore its frequency is close to the nominal $50 \mathrm{~Hz}$. As a result, the computed voltage and current phasors are 
within expected accuracy bands. This can be seen in Fig. 4(a)Fig. 4(d). The small drift in the voltage and current angles is due to the slight variations in the grid frequency during the experiment.

In contrast, Fig. 4(e)-Fig. 4(h), shows the rotor voltage and current phasors, as computed by the rotor's terminal PMU. These values are imprecise because the rotor voltages and currents are at slip frequency, which is approximately $6.67 \mathrm{~Hz}$. The accuracy of the DFIG's internal parameter estimation largely depends on the accuracy of the PMUs. PMU technology design has so far not considered the use of synchrophasors at off-nominal frequencies. The steadystate and dynamic compliance testing of the PMUs (for its calibration) is performed within a range of $\pm 5 \mathrm{~Hz}$ of the nominal frequency. The accuracy of the PMU deteriorates significantly for out-of-band frequencies. At the slip frequency of $6.67 \mathrm{~Hz}$, the Total Vector Error (TVE) of the PMUs is well above the accuracy limit of $1 \%$, as also stated by the standard [10], and hence, these synchrophasors cannot be used for further analysis. Another reason for erroneous estimation of the synchrophasors at the rotor terminal is due to internal filtering of the PMU, which is designed to extract phasors close to $50 \mathrm{~Hz}$ frequency.

However, closely observing the rotor voltage angle computed by the PMU (see Fig. 4(f)), the phase angle shows a slip frequency of about $6.67 \mathrm{~Hz}$. This proves that the phase angle and the frequency calculated by the rotor PMU is within expected values. However, the rotor voltage magnitude (see Fig. 4(e)), is imprecise. The phase angle of the rotor current is also affected due to the harmonics in the rotor currents (see Fig. 4(h)). Hence, the first hypothesis of utilizing only PMUmeasurements for estimating the internal parameters of the DFIG is, thus, falsified.

\section{B. Revised Experimental Setup with PMU at the Stator Ter- minals and DAQ System at the Rotor Terminals}

Due to the limitations of the PMU technology, the experimental setup is revised and a hybrid acquisition system based on a PMU at the stator terminals and a NI-cRIO based DAQ system at the rotor terminals is installed. The DAQ system is configured to provide both the rotor voltage and current waveforms at a resolution of $1 \mathrm{kHz}$. This sampling rate is chosen to accurately sample the harmonic contents of the rotor voltages and currents, due to the switching of the DFIG rotorconverter. The measurements acquired by the DAQ system are time-tagged using NI-9467 GPS module and then are processed in the algorithm, explained in Fig. 5, and analyzed using the LabVIEW graphical interface.

Fig. 6(a)-Fig. 6(b) shows the rotor voltages and currents, which are obtained from the DAQ system. The information received from these waveforms is then used in the algorithm in Fig. 5, to calculate the magnetization, stator leakage, and rotor leakage inductances.

The outline of the algorithm is shown in Fig. 5, which is derived from the equivalent circuit [7] of the DFIG. The algorithm receives synchrophasor measurements from the stator's PMU and point-of-waveform data from the rotor's DAQ system. The DFIG name-plate data consisting of the stator resistance $R_{\mathrm{s}}$, rotor resistance $R_{\mathrm{r}}^{\prime}$, stator turns-ratio $N_{\mathrm{s}}$, and rotor turns-ratio $N_{\mathrm{r}}$, are fed to the algorithm in Fig. 5, in order to calculate the DFIG's parameters (the magnetization and leakage inductances). In addition, the algorithm computes the core losses, core resistance, and magnetization current in real-time. A LabVIEW based graphical user interface (GUI) is developed to monitor the parameters being computed by the algorithm in real-time. The algorithm update rate is set at 50 updates per second, which is a limitation posed by the resolution of the PMU (which is reporting at a rate of $50 \mathrm{msgs} / \mathrm{sec}$ ).

1) No-load Test: During the no-load test of the DFIG, using the vector control, the rotor converter injects the current such that it flows through the rotor leakage reactance and resistance, and the magnetization reactance. Thus, the stator current is negligible. As a result, as depicted in Fig. 5, using the measured rotor voltages and currents, the sum of the magnetization and rotor leakage inductance is obtained. The algorithm also incorporates the function, which calculates the magnetization inductance. However, it requires another set of rotor current and voltage values, in order to compute it. During the offline tests, this is obtained through the shortcircuit/block-rotor test, which is used to compute the rotor leakage reactance (see Section V-B2).

2) Short-Circuit Test: During the short-circuit test of the DFIG, the stator flux becomes stand-still. Hence, the slip of the generator can be regarded as unity (this is similar to a blocked-rotor test of an induction motor). When a shortcircuit is made at the stator terminals of the DFIG, negligible current flows through the magnetization inductance (because the magnetization reactance is much larger than the leakage reactances), and hence, can be neglected. In this case, the equivalent-circuit of the DFIG is represented by the leakage reactances at both the stator and rotor side $\left(X_{\mathrm{sl}}\right.$ and $\left.X_{\mathrm{lr}}^{\prime}\right)$ along with their respective resistances, shown in Fig. 5. As a result, the leakage reactances can be calculated.

Several test cases have been experimentally implemented on the test setup and are recorded. They are explained as follows:

Test case 1: In this scenario, the no-load operation of the DFIG is assessed, where only the the reactive component of the current in the rotor is increased from $5.2 \mathrm{~A}$ to $30 \mathrm{~A}$, in increments of $5 \mathrm{~A}$. In this case, the rotor slowly takes over the magnetization current from the stator. At rotor currents of $25 \mathrm{~A}$, the generator draws zero current from the grid and, thus, the generator is fully magnetized by the rotor. At this instant, stator and the rotor voltages, rotor currents, and rotor power factor angles are measured. These values are then processed in the algorithm in Fig. 5, computing the sum of the rotor leakage inductance and magnetization inductance. Because zero active power (zero current) is delivered to the grid, then the magnetization voltage (thereby the stator voltage) and its position induced by the rotor-current (supplied by the rotor converter) is found. Thus, the sum of the rotor leakage and magnetization 

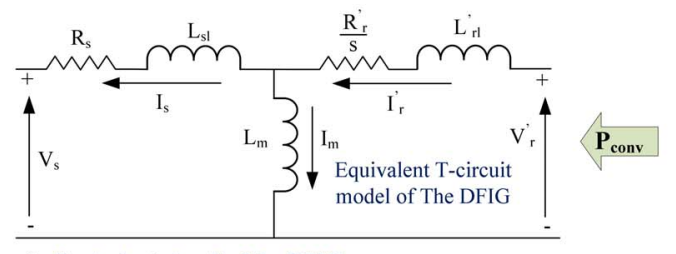

a). Equivalent-circuit of the DFIG

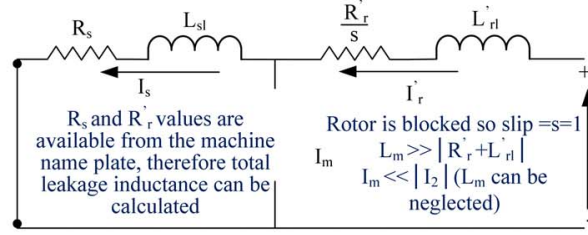

b). Equivalent-circuit of the DFIG during short-circuit test.

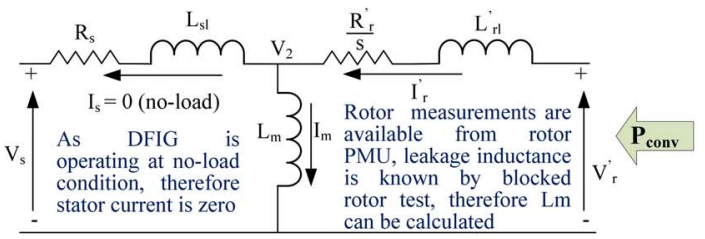

c). Equivalent-circuit of the DFIG during no-load test.

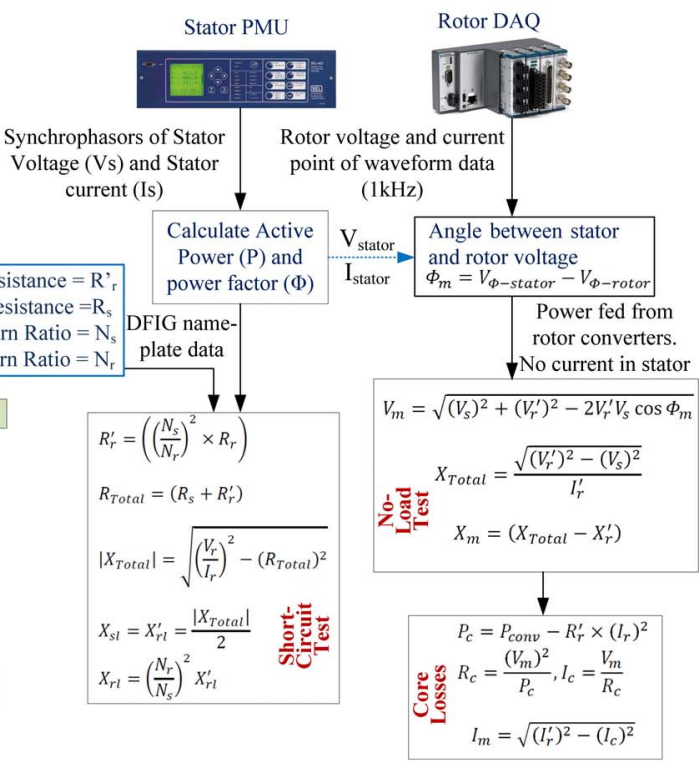

Fig. 5. Flow chart showing the details of the parameter estimation algorithm and the equivalent-circuit of the DFIG.

inductance is estimated. The result is depicted in Fig. 7(a). It is observed that this method computes a quite close estimation of the sum of the rotor leakage and magnetization inductance, as compared with the machine name-plate specifications ${ }^{1}$.

Test case 2: In this scenario, the DFIG rotates at $1700 \mathrm{rpm}$ and delivers $9 \mathrm{~kW}$ power to the grid. The waveforms were captured by the NI-DAQ system when the DFIG is delivering $8 \mathrm{~kW}, 7 \mathrm{~kW}, 6 \mathrm{~kW}$, and $5 \mathrm{~kW}$. These results were then used in the algorithm shown in Fig. 5, which estimates the parameters of the DFIG.

The stator and rotor leakage inductances (total leakage inductance) are shown in Fig. 7(b). It is observed in the Fig. 7(b), that the total stator and rotor leakage inductances cannot be estimated with considerable accuracy, as the values drops to zero at rotor currents of $26 \mathrm{~A}^{2}$. This is because the information is insufficient as the angle between the stator voltage and rotor voltage is unknown. The PMU cannot calculate the angle between them as the two voltages have different frequencies. This demands the study, design, and development of MultiFrequency PMU units, which must be capable of capturing the dynamics of the system at various frequencies.

To deal with this limitation, using the DAQ system, a shortcircuit is applied at the stator terminals, and the rotor voltage and currents are measured. The rotor currents and voltages are then fed to the algorithm, which computes the total leakage reactance, as shown in Fig. 8. It is seen that the algorithm computes the leakage reactances with sufficient accuracy.

\footnotetext{
${ }^{1}$ Note that according to the machine specifications, the magnetization inductance at rated voltage, (i.e., $220 \mathrm{~V}$ per phase) is $23 \mathrm{mH}$, whereas the rotor leakage inductance at rated current, (i.e., $41 \mathrm{~A}$ ) is $0.8 \mathrm{mH}$ ).

${ }^{2}$ Note that according to the machine specifications, the total stator and rotor leakage inductances of the machine at rated current $(41 \mathrm{~A})$ are $2.1 \mathrm{mH})$.
}

If a PMU could calculate the rotor voltage and frequency at the rotor frequency, then the information from it together with the rotor position, can be used in a modified version of the proposed algorithm to compute the total leakage reactances online, without the need of short-circuit tests on the DFIGstator.

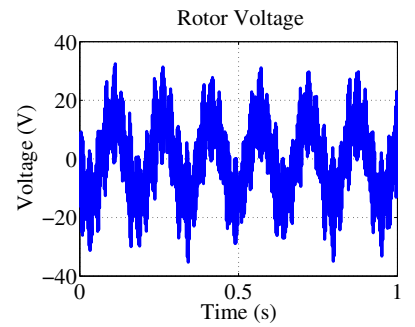

(a)

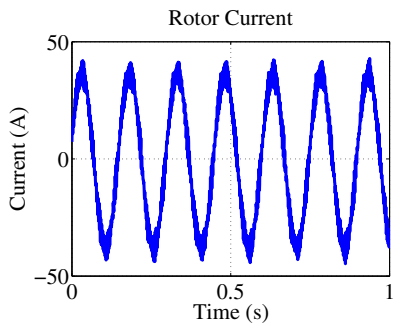

(b)
Fig. 6. Waveforms of the DFIG from the NI-DAQ unit for the: (a) rotor voltage, (b) rotor current.

\section{DISCUSSION}

Commercially available PMUs are designed for nominal frequencies of $50 / 60 \mathrm{~Hz}$ and the reporting of the synchrophasors is limited to $50 / 60 \mathrm{msgs} / \mathrm{sec}$. According to the IEEE C37.118 standard [10], the synchrophasor protocol accommodates time-tagged phasors, digital, and analog quantities. This has been interpreted by PMU vendors as the quantities obtained from internal calculations of PMUs, e.g., active power, reactive power, power factor, etc. Most of the protection relays with PMU features do have additional analog inputs for transducer inputs, e.g., output of the pressure 


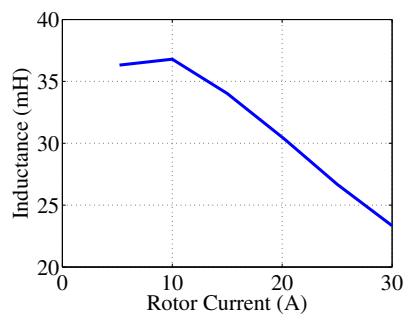

(a)

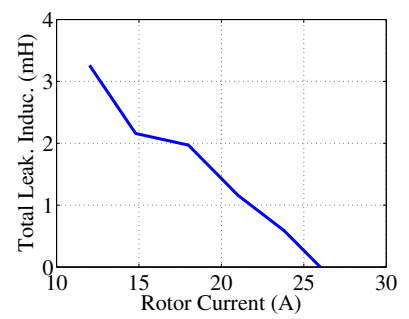

(b)
Fig. 7. Through the stator and rotor PMUs, the real-time estimation of the (a) sum of magnetization and rotor inductance, (b) sum of stator and rotor leakage inductance.

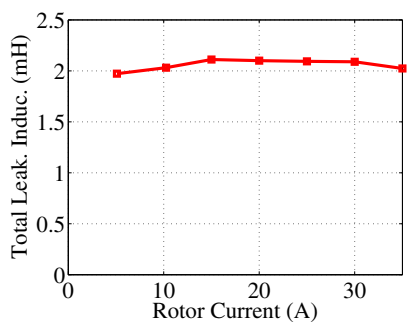

Fig. 8. Offline estimation of the total leakage inductance.

relief valve sensor. However these analog signals are not yet made available in commercial PMUs to be streamed out as synchrophasor analogs, together with the phasor data, with some exceptions in prototypes [12]. If this facility is provided, it will allow addition of the time-tagged waveforms of the rotor voltages and currents having low frequencies, as analogs in the synchrophasor frame. This will expedite the use of synchrophasor data in new potential power system monitoring, protection, and control applications.

In this study, hybrid synchrophasor and point of waveform data is used to estimate the internal parameters of the machine. Though, the resolution of the synchrophasors and point of waveform data were different, (in order to account for the harmonics at the rotor terminal of the DFIG), the experimental results from this paper serve as an evidence, for further research in the development of sub-synchronous time-tagged phasor estimation.

\section{CONCLUSION}

This paper highlighted the current possibilities and limitations of the application of PMU technologies for real-time parameter estimation within plants, i.e., at the basic device level. With the rise in the installation of renewable energy sources, it will become necessary to have synchrophasor data from individual plant equipment for different purposes. For example, accurate estimation of a single unit could provide additional information and confidence on the models used, when developing the aggregate models of the wind-parks that the TSO uses [13]. On the other hand, internal monitoring of the plants connected to the grid via remote control centers, will provide a holistic overview of the power grid, which may become useful during periods of increased production uncertainty.

The results in this paper highlights the need for the development of Multi-Frequency or Dynamic PMU units, so that PMUs may become able to capture multi-frequency dynamics (such as those resulting from the operation of power electronic converters). This will provide better estimation of the parameters of the sub-components of the grid, and enable the implementation of new controllers, that can exploit both local and remote synchrophasors. This paper stresses this point and can be used as a starting point for motivating the practical need for driving further research related to multi-frequency PMUs.

\section{ACKNOWLEDGEMENT}

The authors are grateful to Energimyndigheten, Nordic Energy Research (through the STRONg2rid project), and Statnett SF (the Norwegian Power System Operator) for financial and technical support.

\section{REFERENCES}

[1] S. Chandrasekaran, "Grid connected doubly fed induction generator based wind turbine under LVRT," Doctoral Thesis, University of Bologna, Bologna, Italy, Mar. 2014.

[2] A. Petersson, "Analysis, modeling and control of doubly-fed induction generators for wind turbines," Ph.D. dissertation, Chalmers University of Technology, Goteborg, Sweden, 2005.

[3] Y. He, Y. Feng, and Y. Wang, "Estimating the electrical parameters of induction motors at standstill using rls method," in Proc. 2nd IEEE Int. Symp. on Power Electron. for Distributed Gener. Systems (PEDG), Jun. 2010, pp. 358-363.

[4] Y. Zhang, E. Muljadi, D. Kosterev, and M. Singh, "Wind power plant model validation using synchrophasor measurements at the point of interconnection," IEEE Trans. Sustain. Energy, vol. PP, no. 99, pp. 1-9, Sept. 2014.

[5] V. Terzija, G. Valverde, D. Cai, P. Regulski, V. Madani, J. Fitch, S. Skok, M. Begovic, and A. Phadke, "Wide-area monitoring, protection, and control of future electric power networks," Proceedings of the IEEE, vol. 99, no. 1, pp. 80-93, Jan. 2011.

[6] L. Vanfretti, M. Baudette, and A. White, Monitoring and control of renewable energy sources using synchronized phasor measurements, L. E. Jones, Ed. Elsevier, 2014.

[7] P. C. Krause, O. Wasynczuk, and S. D. Sudhoff, Analysis of Electric Machinery and Drive Systems, 2nd ed. John Wiley and Sons, 2002.

[8] L. Harnefors and H.-P. Nee, "A general algorithm for speed and position estimation of ac motors," IEEE Trans. Ind. Electron., vol. 47, no. 1, pp. 77-83, Feb. 2000.

[9] - "Model-based current control of ac machines using the internal model control method," IEEE Trans. Ind. Appl., vol. 34, no. 1, pp. 133141, Jan. 1998.

[10] "IEEE standard for synchrophasor measurements for power systems," IEEE Std C37.118.1-2011 (Revision of IEEE Std C37.118-2005), pp. 1-61, Dec. 2011.

[11] L. Vanfretti, V. Aarstrand, M. Almas, V. Peric, and J. Gjerde, "A software development toolkit for real-time synchrophasor applications," in Proc. 2013 IEEE Grenoble PowerTech, Jun. 2013, pp. 1-6.

[12] J. Chow, M. Glinkowski, R. Murphy, T. Cease, and N. Kosaka, "Generator and exciter parameter estimation of fort patrick henry hydro unit 1," IEEE Trans. Energy Conv., vol. 14, no. 4, pp. 923-929, Dec. 1999.

[13] L. Vanfretti, T. Bogodorova, and M. Baudette, "Power system model identification exploiting the modelica language and fmi technologies,' in Proc. IEEE Int. Conf. Intelligent Energy and Power Syst. (IEPS), Jun. 2014, pp. 127-132. 